\title{
The Risk Analysis and Modeling of Byco Petroleum in Pakistan Using Extreme Value Theory
}

\author{
Zishan Ali Syed $\mathbb{D},{ }^{1}$ Mohammad Mohammad Ahmed Almazah $\mathbb{D}^{2,3}$ Zahid Iqbal ${ }^{2}{ }^{4}$ \\ and Ghulam Raza Khan $\mathbb{D D}^{5}$ \\ ${ }^{1}$ Govt Associate College Chakri Rawalpindi, Higher Education Department Lahore, Punjab, Pakistan \\ ${ }^{2}$ Department of Mathematics, College of Sciences and Arts (Muhyil), King Khalid University, Muhyil 61421, Saudi Arabia \\ ${ }^{3}$ Department of Mathematics and Computer, College of Sciences, Ibb University, Ibb 70270, Yemen \\ ${ }^{4}$ Department of Statistics, Allama Iqbal Open University, Islamabad, Pakistan \\ ${ }^{5}$ Department of Statistics, University of Wah, Wah Cannt, Rawalpindi, Pakistan
}

Correspondence should be addressed to Ghulam Raza Khan; ghulam.raza@uow.edu.pk

Received 28 July 2021; Accepted 1 September 2021; Published 22 September 2021

Academic Editor: Ishfaq Ahmad

Copyright (c) 2021 Zishan Ali Syed et al. This is an open access article distributed under the Creative Commons Attribution License, which permits unrestricted use, distribution, and reproduction in any medium, provided the original work is properly cited.

\begin{abstract}
The extreme value theory (EVT) has been used to model and measure the distribution of extreme minima of Byco Petroleum in the Pakistan stock market over the period from 2005 to 2012. This paper covers the investigation of distributions that are mostly used in finance including the generalized extreme value (GEV), generalized logistics (GL), and generalized Pareto (GPA) distribution. L-moment ratio diagram is being used to find the appropriate distributions among the distributions. L-moment diagram depicts that GEV and GL distributions are suitable to represent the extremes of Byco Petroleum Pakistan Limited. Thereafter, the probability weighted moment (PWM) method has been used in order to estimate the parameters of probability distributions. Furthermore, Anderson-Darling (AD) goodness-of-fit test is employed to test the goodness of fit among GEV and GL distributions, and it is clear from the results that the GL distribution is more reliable and applicable for extreme minima of Byco Petroleum Company in the Pakistan stock exchange market. EVT and traditional methods are used for value-at-risk (VaR) analysis. The analysis indicates that EVT methods are more suitable for risk measurement in comparison with traditional methods.
\end{abstract}

\section{Introduction}

The insufficiency of risk management was very much obvious after the collapse of the stock market which occurred during the period of 1970s. These crashes affected all the stock markets around the world, especially the oil price shock and fixed exchange rate system. Oil price shock negatively influenced the United States economy. The changes of these prices led to competitive position change in many industries, such as the automobile industry. These events left no room for doubt that effective management of financial risk is inevitable. The crisis did not stop there, but these catastrophes continued in the form of worldwide crisis in 1987, the Mexican crisis in 1995, and the financial crisis of Asia in 1997 which increased the threat of worldwide economic collapse. Orange County, Barings Bank, and Long-Term Capital Management were also some of the notable scenarios of the crisis. By this time, financial crisis, its risks, and the poor financial management were evident. All these issues forced the regulators to address these problems at the earliest. In 1996, the Bank for International Settlements (BIS) amended the Capital Accord of 1988 to save from drastic price risk. This amendment also forced the qualitative standard to improve risk management techniques for all the financial markets. It presented the model of value at risk (VaR) for estimating the capital adequacy requirements (CARs). The basic purpose of the CAR was to cover the possible loss that results from market position and help to fight financial crisis (Aboura [1]). 
VaR helps to examine the loss at different confidence levels covering a specific time period. It can be termed as the lower quantile of distribution that does not cross the certain limit. According to Ahmed Ghorbel [2], there are three traditional methods that are used to calculate VaR: the variance-covariance (VC), the historical simulation (HS), and the Monte Carlo simulation (MCS). The VC method depends on the thought that financial returns have normal distribution, but other research studies set forth that they have fatter tails than normal distribution. The presumption is that the probability of loss is more than suggested by the normal distribution. Conversely, the HS method does not need to infer returns of distributions in order to find VaR. It only delineates that historical returns can be good to predicate future returns, but this method lags behind because of the insufficiency of data which can lead to unreliable results. MCS can be used to calculate $\mathrm{VaR}$, but it needs more computing skills to bring about results.

Even though $\mathrm{VaR}$ is being widely used, still there is no concordance among professionals and scholars for which method can be best in calculating VaR. The common flaw of the traditional method is that it does not rightly manipulate lower tails of distribution. It is unable to give right results when we need them the most (Evis Kellezi [3]).

On the contrary, EVT is the branch of the statistics which deals with the extremes to asses a given random variable to find the probability in events which are more extreme than the last observation. EVT-based models are specially designed for extreme events. It provides the models with which we can represent the tails of distribution. The theory has been applied in many areas, for instance, engineering and hydrology, and after that, areas of finance and insurance showed increased interest in it. The application of EVT is not an old development but flourished instantaneously (Davison and Smith [4]).

The pioneer to use EVT in finance was Parkinson [5]. He used extreme returns' data that were available to compare the variance of estimated returns. He postulated that the extreme value method can render much better evaluation of variance than the old methods. Another leading name in putting forward the EVT in finance was no doubt Longin. Longin examined the distribution of extremes in the stock market of USA [6]. He studied the extreme movement of the US stock market containing the daily observations of over hundred years (1885-1990). These extreme variables depend on the returns distributed and the selected time period. Empirically, Longin and Felipe [6] posed that minima and maxima could be accurately characterized by the Frechet distribution. Frechet distribution is an important occurrence in GEV distribution. Michael Rockinger [7] gave the international extreme value theory application. They used the data of stock markets for about 20 countries. They evaluate congruence in right and left tails of returns, all across countries. They used EVT to show that the left tail returns are the same as the right tail returns and concluded that this phenomenon is not the result of clustering of extremes. They also established the point that GP gave good fit to the experimental dataset. Withal, they rationalized extreme value theory (EVT) for the estimation of $\mathrm{VaR}$, but it is not as good as it could not figure out the S\&P 500 index large negative daily returns of $-22.83 \%$ in October.

The methods that are being used in this paper were also implied to European stock markets. Lux [8] manipulated the data sample of the DAX index covering the period from 1988 to 1994. He posed the thought that the tails of distribution are thinner using Pareto distribution. He evaluated the German stock market data by fitting the GEV distribution and concluded that Frechet distribution is good to fit in maximum occurrences. He also found the same results on DAX index returns implying intraday data.

Another important step was the application of EVT in estimating VaR. This has been tested and put forward by Pownall and Koedijk [9]. They collected the dataset of Asian stock markets and made the comparison of $\mathrm{VaR}$ computation by normal distributions. They also used the RiskMetrics model of Morgan et al. [10] with evaluation originated by EVT. They concluded that VaR estimated apparently better than other models and techniques.

In EVT, GEV and GP are thought to be the only techniques which can fit extremes (minima and maxima). In recent studies, Gettinby et al. [11] delineated the distribution of extremes. For this, they used the data of shares of an index of UK consisting of the time period from 1975 to 2000. They examined the number of distributions: GEV, Frechet, GP, Weibull, and GL. They examined and compared each distribution to find the best fit. They evaluated the extremes of the UK index with each of the distributions separately. They concluded that, empirically, GL fits the minima and maxima. GL gives better outcomes as compared to GEV.

Another work that seconds Gettinby et al.'s study was recently put forward by Tolikas and Brown [12]. They also presented generalized logistics (which is not given attention as much as GEV and GP) when it comes to the EVT application. Despite, it gives more stable results for Athens Stock Exchange. They gathered the data of ASE of the period from 1986 to 2001 and found the minimum daily returns applying the EVT method. Hence, empirically, they concluded GL distribution to be far better than GEV and GP, and GL has fatter tails than GEV. Similarly, the application of EVT in the field of biostatistics is given in [13-15].

In this paper, we mainly focused on the measurement of the extreme risk of Byco Petroleum Company in the Pakistan Stock Market. We collected the dataset of Byco Petroleum Pakistan covering the time span of 8 years from 2005 to 2012. We found the daily, weekly, and monthly logarithmic returns of chosen samples of that dataset. After finding logarithmic returns, two distributions were fitted to these returns. L-moment ratio diagram was the technique to identify the distribution of the extreme minimum daily returns. These distributions were GEV and GL. AD goodness-of-fit test was used to test the goodness of fit. Findings of this paper indicate that GL is a better fit for these returns. 


\section{Methodology}

2.1. Extreme Value Theory. EVT is a branch of statistics which deals with the extreme observation. The main role of EVT is to empirically estimate and determine the extreme behavior of random variables. According to Longin and Felipe [6], extreme observations are either minimum or maximum of returns of certain intervals such as daily, weekly, and monthly. For a more clear understanding, let us assume variables $Y_{1}, Y_{2}$, $\ldots, Y_{n}$ as the time series of the daily index and denote the extreme minima with $X_{1}=\min \left(Y_{1}, \ldots, Y_{m}\right), X_{2}=\min \left(Y_{m+1}\right.$, $\left.\ldots, Y_{2 m}\right), \ldots, \ldots, X_{n / m}=\min \left(Y_{n-m}, \ldots, Y_{n}\right)$. Now, the point here is to acquire the adequate probability distribution which will assess and explain the behavior of minimum $X_{1}, X_{2}, \ldots$, $X_{n / m}$.

According to presupposition, returns are independent and identically distributed (iid). Gnedenko [16] based his study on this thought: he explained that the generalized extreme value distribution is a limiting distribution of extremes. GEV holds huge support theoretically, but there is also great backing to the thought that financial returns show serial correlation and heteroscedasticity. Kearns and Pagan [17] also underlay their study on iid assumption. They used the Monte Carlo simulation (MCS) technique to present that violating the iid assumption exaggerates the shape parameter estimates. Leadbetter et al. [18] examined the extreme value theory, and findings of their paper showed that it can deal with weak data structures. Jondeau and Rockinger [19] gathered the data of DAX index daily returns covering the period of 1969 to 1998 . They analyzed the extremes of German stock market. They also examined time series returns for autocorrelation and volatility. The finding of the paper showed no significant change in results, so the analysis can be performed without the iid assumption. GL and other distributions were also considered for empirical applications.

Logarithmic returns are divided into daily, weekly, and monthly intervals. Daily extremes are divided into 10 and 30 subperiods, weekly into 2, 4, and 10 subperiods, and monthly into 2 subperiods.

After dividing the extremes into subperiods of daily, weekly, and monthly, further job is to look for the suitable distributions for the data. Those distributions will be considered for analysis which can represent fat tail. GEV, GL, and GP were selected because of the influence of flood frequency analysis. These distributions deal with the three parameters. These three parameters are location $(\alpha)$, scale $(\beta)$, and shape $(\gamma)$. The first parameter location represents mean; the higher value of mean signifies the larger extremes. The second parameter scale corresponds to standard deviation. High value of standard deviation shows that the distribution of extremes is largely spread. The third parameter shape is the most important parameter of all because a higher value of the shape parameter is analogous to fatter tails. In the frame reference of finance, it means that high values of the shape parameter depict large negative returns or heightened probability of crash (Aparicio and Estrada [20]).
L-moments are used to identify the suitable distribution of all the three chosen distributions. L-moment estimation technique was developed by Hosking [21]. This technique is widely used because one can identify the best fitted distribution and obtain more accurate parametric estimates as compared to conventional moment estimators.

In the next step, probability weighted moment method is used to estimate the parameter of distributions. In this approach, sample moments of GL and GEV are equated to estimate parameters. Hosking and Wallis [22] gave an easy description of PWM. Let $X$ be the random variable having finite mean and $F$ be the distribution function; then,

$$
\alpha_{r}=E\left[X\{1-(X)\}^{r}\right], \quad r=1,2, \ldots
$$

PWM is useful while dealing with small samples of data because extremes are not high even in large samples. Hosking established the view that robust L-moment and PWM have a linear relationship, which is shown in four equations of L-moments.

$$
\begin{aligned}
& \text { (i) } \lambda_{1}=E(X)=\alpha_{0} \\
& \text { (ii) } \lambda_{2}=1 / 2 E\left(\left(X_{2: 2}\right)-\left(X_{1: 2}\right)\right)=\alpha_{0}-2 \alpha_{1} \\
& \text { (iii) } \lambda_{3}=1 / 3 E\left(\left(X_{3: 3}\right) 2\left(X_{2: 3}\right)+\left(X_{1: 3}\right)\right)=\alpha_{0}-6 \\
& \quad \alpha_{1}+6 \alpha_{2} \\
& \text { (iv) } \lambda_{4}=1 / 4 E\left(\left(X_{4: 4}\right)-3\left(X_{3: 4}\right)+3\left(X_{2: 4}\right)-\left(X_{1: 4}\right)\right) \\
& =\alpha_{0}-12 \alpha_{1}+30 \alpha_{2}-20 \alpha_{3}
\end{aligned}
$$

The next step after estimating parameters is to know which distribution is the best fit. Anderson-Darling test is used for this purpose. Anderson-Darling test statistics are defined by

$$
\int_{-\infty}^{\infty}\left[F_{n}(x)-F(x)\right]^{2} \varnothing(x) \mathrm{d} F(x)
$$

where $F(x)$ is the distribution function of $X$ and $n$ is the number of observations.

At last, after properly modeling the distributions of extremes, lower quantiles have been put to use in order to calculate VaR estimates. As the length of minima is specified of certain time period, confidence level is adjusted to obtain the desired frequency of EVT-based VaR. Probability of extreme returns is denoted by $p_{\text {ext }}$. It is presumed that collected daily returns will not exceed $\mathrm{VaR}$. The probability of extreme returns is

$$
p_{\text {ext }}=(1-p)^{T}
$$

$\mathrm{VaR}$ is thought to be effective only when it provides accurate results, and to know its efficiency, these models are needed to be validated. Backtesting can be used for the validation of $\mathrm{VaR}$ models. $\mathrm{VaR}$ is rightly specified when the confidence level is equal to the violations of returns. For VaR validation, we employed Christoffersen [23] test.

\section{Data Description}

In this paper, we used the dataset consisting of 1920 daily logarithmic returns collected from the data stream. The data 
were taken from the State Bank of Pakistan and cover the 8year period from January 3, 2005, to December 21, 2012.

In Table 1, the Byco Petroleum daily returns, negative skewness of -1.07 , and kurtosis 11.22 can be seen. It clearly demonstrates that a large number of daily returns deviate from normality. This deviation from normality was also assessed by the Shapiro-Wilk test. It is clear that both subperiods deviated from normality, but the second subperiod was more volatile in comparison with the first subperiod.

In Table 2, $\mu$ is denoted as mean and $\sigma$ is the standard deviation. Expected frequency represents large negative daily returns which exceeded the defined threshold. Total values are divided into two subperiods. It also contains the values expected on the normality assumption. In cluster row includes the values of daily returns preceding another daily return within two trading weeks.

Daily returns of Byco Petroleum are examined as shown in Table 2 in which the main focus is on the left tail distribution. In the table, $\mu$ is denoted as the overall mean, and $\sigma$ is denoted as the overall standard deviation. Frequency of the negative daily returns exceeded all three thresholds: $\mu-2 \sigma, \mu-3 \sigma$, and $\mu-4 \sigma$. The result of the table showed that all the daily returns which were above the limit of the threshold were also higher than assumed normality.

After finding values above the threshold, the next job was to look for the tendency to cluster of these returns with the passage of time. For this purpose, returns were computed with their lower and higher values of a certain time period of two weeks. It can be noticed that clustering heightened under the first threshold $\mu-2 \sigma$. The table showed that the investors who will take this normal distribution as a base while investing in Pakistan Stock Market will not be able to accurately foresee the risk.

On the whole, descriptive statistics demonstrate that the distribution of daily returns is different in two subperiods examined.

\section{Analysis of the Extremes in the Pakistan Stock Market}

Byco Petroleum daily index covering the period of 8 years is collected to find the daily, weekly, and monthly minima. Table 1 shows the descriptive statistics of minima. It clearly illustrated that mean minima increased as we increased the interval, and all subperiods deviated from normality.

\subsection{Identification of the Distribution of the Extreme Minimum} Daily Returns Using the L-Moment Diagram. L-moment diagram is used to determine the suitable distribution for extreme daily returns. Using the data of daily minima, L-skewness and L-kurtosis were calculated for 30 subperiods of daily returns. Then, these calculations of L-skewness and L-kurtosis were plotted on the L-moment diagram. In the diagram, it can be seen that $\mathrm{T}_{3}$ and $\mathrm{T}_{4}$ were divided into the series of 30 subperiods for daily minima. One can clearly infer in the first glance that GL and GEV are only suitable distributions excluding other distributions because all the points of $\tau_{3}$ and ${ }_{\tau_{4}}$ lie around the curves of GL and GEV. Despite all, L-moment does not clearly determine which distribution is to be focused among GL and GEV. For this purpose, these two distributions need to be analyzed by the goodness-of-fit test.

L-moment ratio diagram for the daily returns of Byco Petroleum Pakistan Limited indicates that the L-skewness and L-kurtosis are mainly concentrated around the theoretical curves of the generalized logistics and generalized extreme value distribution indicating that these two distributions are likely to adequately fit the empirical data.

4.2. Parameter Estimates and Goodness-of-Fit Test. GL and GEV distributions were used to fit the whole sample and subperiods of daily, weekly, and monthly minima of Byco Petroleum Pakistan Limited. After that, using probability weighted moment (PWM), parameters of GL and GEV distributions were calculated for all the samples and subperiods of daily, weekly, and monthly minima. Then, $p$ values of $\mathrm{AD}$ goodness-of-fit test were found as shown in Tables 3-5. GL and GEV distributions were now fitted to the whole sample, in which the $p$ value indicated that both distributions do not give adequate description, but when we divided the data into subperiods, both GEV and GL provided an adequate description. For example, when we used 10 subperiods, 7 subperiods showed adequate results; however, GL performed better than GEV. When we used 30 subperiods, GL provided better results in 27 subperiods, and the GEV provided adequate results in 24 subperiods. The GL gave better results as compared to GEV in 23 subperiods and GEV in 6 of them.

The $\mathrm{AD} p$ value for the GEV distribution ranges from 0.000 to 0.774 , and the $p$ value for the GL distribution ranges from 0.002 to 0.940 .

In the GEV distribution, the shape parameter sometimes takes positive values which signify Weibull distribution, whereas negative values go for Frechet. Previous studies [6] in the USA and [11] in the UK showed no change of sign in the shape parameter. In Figure 1, it can be noticed that the shape parameter fitted with GL and GEV was stable in the first half and greatly variable in the second half. The variability of the shape parameter in the second half can affect VaR estimates significantly.

In Table 3, the probability weighted moment estimates and Anderson-Darling $p$ value of GEV and GL distributions for the daily minima returns of Byco Petroleum Pakistan Limited were calculated. $N$ denotes the number of extreme observations in each period. Alpha denotes the location parameter, beta denotes the scale parameter, and gamma denotes the shape parameter.

Figure 2 illustrates the behavior of shape parameter estimates of GL and GEV distributions for the daily minima divided over 30 subperiods.

In Figure 2, it can be noticed that the shape parameter fitted with GL and GEV was stable in the first half and greatly variable in the second half. The variability of the shape parameter in the second half can affect VaR estimates significantly. Higher values of the shape parameter can lead to 
Table 1: Descriptive statistics for Byco Petroleum Company daily returns.

\begin{tabular}{|c|c|c|c|c|c|c|c|c|c|}
\hline & $N$ & $m$ & Mean & St. dev & Min & Max & Skewness & Kurtosis & SW \\
\hline \multicolumn{10}{|l|}{ 2005-2012 } \\
\hline Daily minima & 1920 & & 0.02 & 0.04 & -0.19 & 0.32 & -1.07 & 11.22 & 0.8675 \\
\hline Weekly & 384 & 5 & 0.03 & 0.08 & -0.77 & 0.48 & -1.41 & 20.31 & 0.8349 \\
\hline Monthly & 97 & 20 & 0.01 & 0.15 & -0.9 & 0.04 & -2.01 & 10.23 & 0.8570 \\
\hline \multicolumn{10}{|l|}{$2005-2008.5$} \\
\hline Daily & 959 & & 0.01 & 0.04 & -0.19 & 0.28 & 0.49 & 8.31 & 0.8936 \\
\hline Weekly & 191 & 5 & -0.02 & 0.01 & 0.77 & 0.49 & -1.57 & 18.99 & 0.8049 \\
\hline Monthly & 48 & 20 & 0.03 & 0.19 & 0.9 & 0.35 & -1.83 & 6.72 & 0.8587 \\
\hline \multicolumn{10}{|l|}{$2008.5-2012$} \\
\hline Daily & 960 & & 0.01 & 0.03 & -0.13 & 0.32 & 2.03 & 15.64 & 0.8314 \\
\hline Weekly & 192 & 5 & 0.04 & 0.06 & -0.25 & 0.22 & -0.09 & 2.8 & 0.9319 \\
\hline Monthly & 48 & 20 & 0.01 & 0.01 & 0.21 & 0.25 & 0 & 0.4 & 0.9759 \\
\hline
\end{tabular}

Table 2: Frequency of Byco Petroleum Company index large negative daily returns.

\begin{tabular}{|c|c|c|c|}
\hline Threshold & $<\mu-2 \sigma$ & $<\mu-3 \sigma$ & $<\mu-4 \sigma$ \\
\hline \multicolumn{4}{|l|}{$2005-2012$} \\
\hline Total & 102 & 45 & 15 \\
\hline Expected on the assumption of normality & 70 & 15 & 0 \\
\hline In cluster & 55 & 20 & 8 \\
\hline \multicolumn{4}{|l|}{$2005-2008.5$} \\
\hline Total & 52 & 25 & 7 \\
\hline Expected on the assumption of normality & 34 & 8 & 0 \\
\hline In cluster & 25 & 10 & 5 \\
\hline \multicolumn{4}{|l|}{$2008.5-2012$} \\
\hline Total & 50 & 20 & 8 \\
\hline Expected on the base of normality & 36 & 7 & 0 \\
\hline In cluster & 30 & 10 & 3 \\
\hline
\end{tabular}

TABLE 3: The probability weighted moment estimates and AD p value of GEV and GL distributions for the daily minima returns of Byco Petroleum Pakistan Limited.

\begin{tabular}{|c|c|c|c|c|c|c|c|c|c|}
\hline \multicolumn{5}{|c|}{ GEV estimates } & \multicolumn{5}{|c|}{ GL estimates } \\
\hline$N$ & Alpha & Beta & Gamma & $p$ value & Alpha & Beta & Gamma & $p$ value & Better fit \\
\hline \multicolumn{10}{|l|}{$S=1$} \\
\hline 1920 & -0.01217 & 0.0288 & 0.1951 & $p \leq 0.001$ & -0.0016 & 0.0171 & -0.0505 & 0.022 & GL \\
\hline \multicolumn{10}{|l|}{$S=10$} \\
\hline 192 & -0.0133 & 0.0263 & 0.1411 & 0.701 & -0.0036 & 0.0160 & -0.0823 & 0.923 & GL \\
\hline 192 & -0.0122 & 0.0356 & 0.2507 & 0.742 & -0.0013 & 0.0205 & -0.0185 & 0.760 & GL \\
\hline 192 & -0.0110 & 0.0212 & 0.1923 & 0.556 & -0.0033 & 0.0126 & -0.0521 & 0.810 & GL \\
\hline 192 & -0.0085 & 0.0270 & 0.2444 & 0.678 & 0.0011 & 0.0156 & -0.0221 & 0.761 & GL \\
\hline 192 & -0.0234 & 0.0515 & 0.2973 & 0.221 & -0.0054 & 0.0289 & 0.0075 & 0.050 & GEV \\
\hline 192 & -0.0129 & 0.0292 & 0.0961 & 0.123 & -0.0019 & 0.0183 & -0.1096 & 0.261 & GL \\
\hline 192 & -0.0155 & 0.0305 & 0.0487 & 0.334 & -0.0038 & 0.0196 & -0.1389 & 0.034 & GL \\
\hline 192 & -0.0077 & 0.0179 & 0.1923 & 0.702 & -0.0012 & 0.0106 & -0.0520 & 0.380 & GEV \\
\hline 192 & -0.0074 & 0.0207 & 0.3197 & 0.536 & -0.0001 & 0.0115 & 0.0198 & 0.231 & GL \\
\hline 192 & -0.0091 & 0.0235 & 0.3303 & 0.159 & -0.0004 & 0.0453 & -0.0944 & 0.615 & GEV \\
\hline \multicolumn{10}{|l|}{$S=30$} \\
\hline 64 & -0.0148 & 0.0280 & 0.2070 & 0.325 & -0.0047 & 0.0165 & -0.0435 & 0.910 & GL \\
\hline 64 & -0.0155 & 0.0267 & 0.1347 & 0.576 & -0.0056 & 0.0163 & -0.0861 & 0.443 & GEV \\
\hline 64 & -0.0094 & 0.0243 & 0.0989 & 0.062 & -0.0002 & 0.0152 & -0.1078 & 0.552 & GL \\
\hline 64 & -0.0074 & 0.0332 & 0.1679 & 0.771 & 0.0047 & 0.0200 & -0.0664 & 0.916 & GL \\
\hline 64 & -0.0096 & 0.0365 & 0.2540 & 0.774 & 0.0033 & 0.0210 & -0.0166 & 0.940 & GL \\
\hline 64 & -0.0167 & 0.0366 & 0.3184 & 0.738 & -0.0040 & 0.0204 & 0.0191 & 0.820 & GL \\
\hline 64 & -0.0091 & 0.0187 & 0.2865 & 0.070 & -0.0025 & 0.0106 & 0.0015 & 0.412 & GL \\
\hline 64 & -0.0116 & 0.0199 & 0.0979 & 0.701 & -0.0041 & 0.0124 & -0.1084 & 0.547 & GEV \\
\hline 64 & -0.0125 & 0.0251 & 0.2088 & 0.117 & -0.0034 & 0.0148 & -0.0425 & 0.326 & GL \\
\hline 64 & -0.0658 & 0.0262 & 0.2381 & 0.758 & 0.0038 & 0.0169 & -0.0256 & 0.867 & GL \\
\hline
\end{tabular}


TABLE 3: Continued.

\begin{tabular}{|c|c|c|c|c|c|c|c|c|c|}
\hline \multicolumn{5}{|c|}{ GEV estimates } & \multicolumn{5}{|c|}{ GL estimates } \\
\hline$N$ & Alpha & Beta & Gamma & $p$ value & Alpha & Beta & Gamma & $p$ value & Better fit \\
\hline 64 & -0.0112 & 0.0261 & 0.2106 & 0.391 & -0.0017 & 0.0153 & -0.0415 & 0.800 & GL \\
\hline 64 & -0.0077 & 0.0292 & 0.2988 & 0.682 & 0.0014 & 0.0147 & 0.0083 & 0.665 & GEV \\
\hline 64 & -0.0096 & 0.0182 & 0.3900 & 0.439 & -0.0034 & 0.0098 & -0.0576 & 0.510 & GL \\
\hline 64 & -0.0258 & 0.0484 & 0.2559 & 0.201 & -0.0087 & 0.0276 & -0.0155 & 0.113 & GEV \\
\hline 64 & -0.0349 & 0.0797 & 0.2471 & 0.767 & -0.0064 & 0.0460 & -0.0205 & 0.887 & GL \\
\hline 64 & -0.0224 & 0.0457 & 0.0549 & 0.229 & -0.0050 & 0.0293 & -0.1351 & 0.002 & GL \\
\hline 64 & -0.0076 & 0.0166 & 0.1127 & 0.248 & -0.0014 & 0.0103 & -0.0994 & 0.270 & GL \\
\hline 64 & -0.0086 & 0.0212 & 0.0657 & 0.002 & -0.0006 & 0.0135 & -0.1283 & 0.030 & GL \\
\hline 64 & -0.0138 & 0.0250 & -0.1336 & 0.771 & -0.0038 & 0.0178 & -0.2587 & 0.911 & GL \\
\hline 64 & -0.0155 & 0.0292 & 0.2120 & 0.001 & -0.0050 & 0.0293 & -0.1351 & 0.002 & GL \\
\hline 64 & -0.0165 & 0.0370 & 0.0971 & 0.274 & -0.0026 & 0.0232 & -0.1089 & 0.630 & GL \\
\hline 64 & -0.0046 & 0.0176 & 0.2146 & 0.178 & 0.0016 & 0.0103 & -0.0391 & 0.821 & GEV \\
\hline 64 & -0.0093 & 0.0180 & 0.2571 & 0.247 & -0.0020 & 0.0103 & -0.0149 & 0.467 & GL \\
\hline 64 & -0.0090 & 0.0184 & 0.1384 & 0.750 & -0.0037 & 0.0112 & -0.0840 & 0.308 & GL \\
\hline 64 & -0.0095 & 0.0240 & 0.4561 & 0.310 & -0.0008 & 0.0124 & 0.0918 & 0.096 & GL \\
\hline 64 & -0.0078 & 0.0197 & 0.3811 & 0.200 & -0.0019 & 0.0111 & -0.0985 & 0.400 & GL \\
\hline 64 & -0.0168 & 0.0347 & 0.0911 & 0.034 & -0.0029 & 0.0218 & -0.1127 & 0.048 & GL \\
\hline 64 & -0.0057 & 0.0133 & 0.1996 & 0.160 & -0.0021 & 0.0078 & -0.0478 & 0.905 & GEV \\
\hline 64 & -0.0050 & 0.0181 & -0.0122 & 0.567 & -0.0016 & 0.0120 & -0.1784 & 0.825 & GEV \\
\hline 64 & -0.0046 & 0.0179 & 0.1142 & 0.302 & -0.0011 & 0.0106 & 0.0528 & 0.309 & GL \\
\hline
\end{tabular}

TABle 4: Weekly minima GEV and GL PWM parameter estimates and AD $p$ value.

\begin{tabular}{|c|c|c|c|c|c|c|c|c|c|c|}
\hline \multicolumn{6}{|c|}{ GEV estimates } & \multicolumn{5}{|c|}{ GL estimates } \\
\hline Subperiods & $N$ & Alpha & Beta & Gamma & $p$ value & Alpha & Beta & Gamma & $p$ value & Better fit \\
\hline \multicolumn{11}{|l|}{$S=1$} \\
\hline & 384 & -0.0249 & 0.0732 & 0.3339 & 0.136 & -0.0003 & 0.0403 & 0.02757 & 0.039 & GEV \\
\hline \multicolumn{11}{|l|}{$S=2$} \\
\hline & 192 & -0.0323 & 0.0864 & 0.3617 & 0.338 & -0.0028 & 0.0470 & 0.0425 & 0.185 & GEV \\
\hline & 192 & -0.0183 & 0.0582 & 0.2578 & 0.035 & 0.0023 & 0.0334 & -0.1456 & 0.149 & GL \\
\hline \multicolumn{11}{|l|}{$S=4$} \\
\hline & 96 & -0.0319 & 0.0705 & 0.1735 & 0.696 & -0.0061 & 0.0423 & -0.0631 & 0.335 & GEV \\
\hline & 96 & -0.0314 & 0.1003 & 0.5300 & 0.029 & 0.0007 & 0.0562 & 0.1288 & 0.069 & GL \\
\hline & 96 & -0.0174 & 0.0633 & 0.2624 & 0.455 & 0.0050 & 0.0362 & -0.0119 & 0.520 & GL \\
\hline & 96 & -0.0197 & 0.0536 & 0.2432 & 0.160 & -0.0006 & 0.0308 & -0.0171 & 0.160 & GL \\
\hline \multicolumn{11}{|l|}{$S=10$} \\
\hline & 38 & -0.0286 & 0.0710 & 0.3456 & 0.610 & -0.0041 & 0.0389 & 0.0338 & 0.435 & GEV \\
\hline & 38 & -0.0356 & 0.0824 & 0.0425 & 0.640 & -0.0024 & 0.0532 & -0.1428 & 0.460 & GEV \\
\hline & 38 & -0.0246 & 0.0587 & 0.4581 & 0.589 & -0.0053 & 0.0304 & 0.0928 & 0.367 & GEV \\
\hline & 38 & -0.0034 & 0.0615 & 0.5681 & 0.009 & 0.0160 & 0.0302 & 0.1473 & 0.165 & GL \\
\hline & 38 & -0.0661 & 0.1445 & 0.5422 & 0.330 & -0.0198 & 0.0724 & 0.1347 & 0.178 & GEV \\
\hline & 38 & -0.0140 & 0.0592 & 0.2419 & 0.210 & 0.0071 & 0.0342 & -0.0235 & 0.289 & GL \\
\hline & 38 & -0.0226 & 0.0855 & 0.2170 & 0.035 & 0.0081 & 0.0502 & 0.0378 & 0.066 & $\mathrm{GL}$ \\
\hline & 38 & 0.0217 & 0.0393 & 0.2585 & 0.164 & -0.0077 & 0.0225 & -0.0141 & 0.106 & GEV \\
\hline & 38 & -0.0186 & 0.0572 & 0.4396 & 0.052 & 0.0003 & 0.0299 & 0.0834 & 0.063 & GL \\
\hline & 38 & -0.0130 & 0.5508 & 0.2001 & 0.936 & 0.0069 & 0.0326 & -0.0475 & 0.936 & GL \\
\hline
\end{tabular}

TABle 5: Monthly minima GEV and GL PWM parameter estimates and AD test.

\begin{tabular}{lccccccccccc}
\hline Subperiod & $N$ & Alpha & Beta & Gamma & $p$ value & Alpha & Beta & Gamma & $p$ value & Better fit \\
\hline$S=1$ & & & & & & & & & \\
& 96 & -0.0368 & 0.1488 & -0.5397 & 0.310 & 0.0106 & 0.0742 & 0.1335 & 0.340 & GL \\
\hline$S=2$ & & & & & & & & & & \\
& 48 & -0.0584 & 0.1915 & 0.6218 & 0.270 & 0.0006 & 0.0919 & 0.1728 & 0.261 & GEV \\
& 48 & -0.0197 & 0.0984 & 0.2913 & 0.721 & 0.0148 & 0.0555 & 0.0041 & 0.800 & GL \\
\hline
\end{tabular}




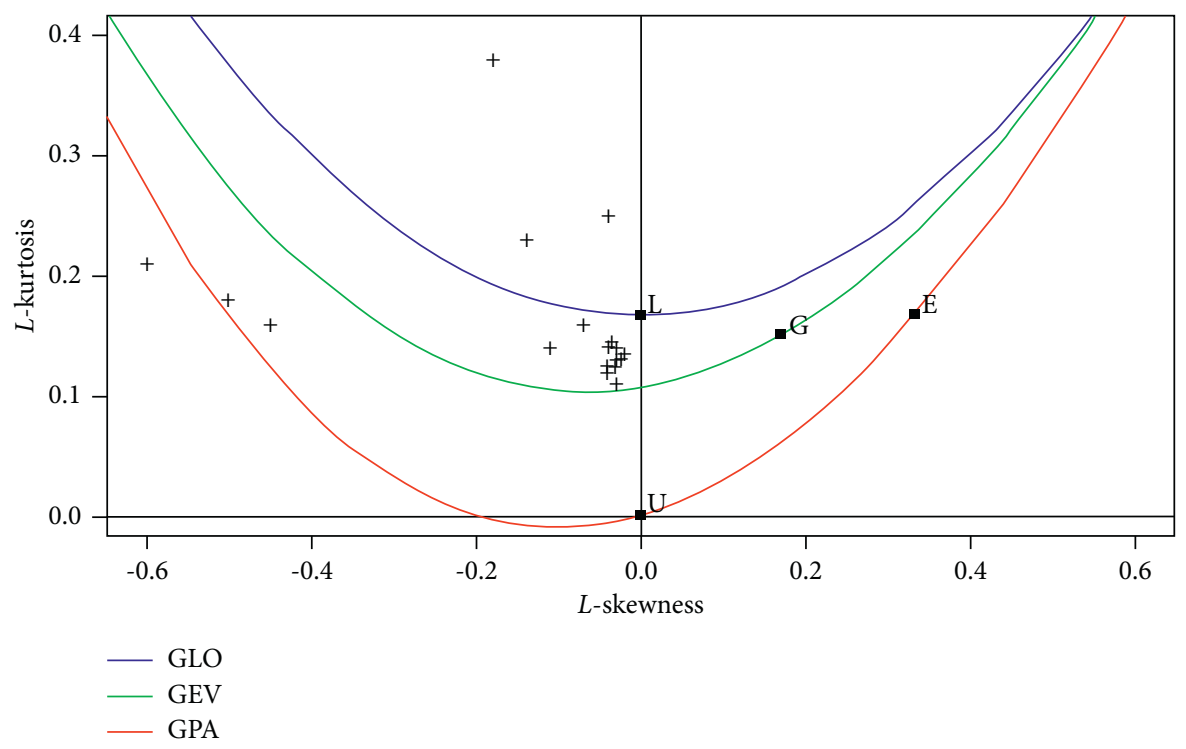

Figure 1: L-moment ratio diagram for the daily returns of Byco Petroleum Pakistan Limited.

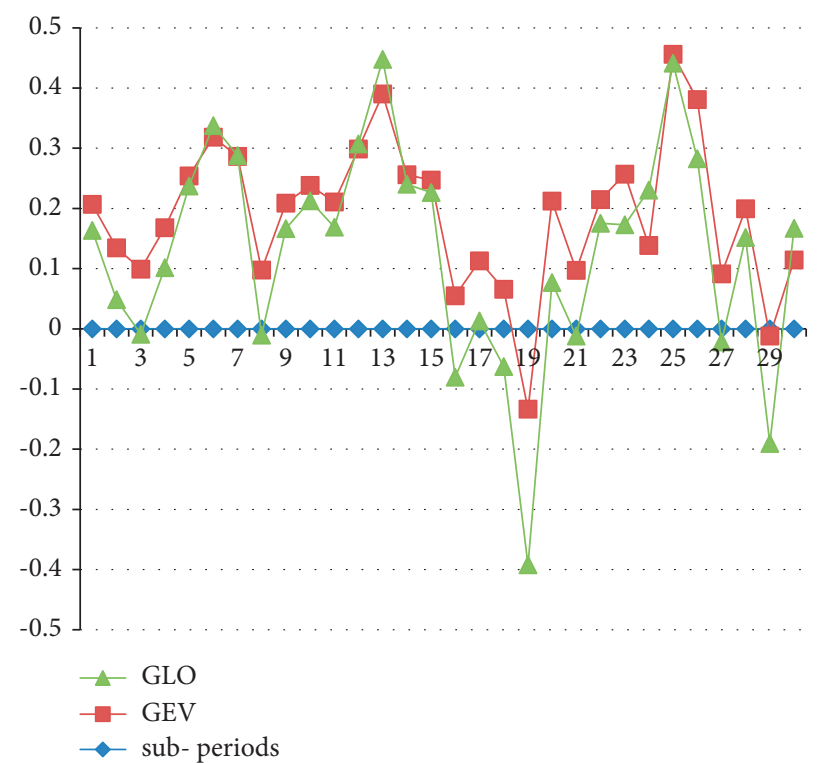

Figure 2: Shape parameter estimates for GEV and GLO.

higher estimation of the value at risk. The whole series of extremes and the series of subperiods were fitted to GL and GEV in order to evaluate the variability of extremes.

Table 4 illustrates the findings of weekly minima of the whole period. It showed that GEV gave the adequate fit with the $p$ value of 0.136 . On the contrary, GL could not prove to be a good fit for the series of weekly minima of the whole period, but when the subperiods of weekly minima were fitted to GL and GEV, GL proved to be a better fit.

In Table 5, the PWM estimates and AD $p$ value for GEV and GL distributions for the weekly minima returns of Byco Petroleum Pakistan were calculated. $N$ denotes the number of extreme observations in each period. Alpha denotes the location parameter, beta denotes the scale parameter, and gamma denotes the shape parameter.

We examined the behavior of stock exchange extremes by fitting the whole data and subperiods of Byco Petroleum Pakistan Limited. The results of both GEV and GL distributions were presented in the tables with the $\mathrm{AD} p$ value. When fitted to the daily extremes, the GL distribution shows the better fit, while for the subperiods, it showed a mixed behavior; sometimes, it is GEV which showed the better fit, and sometimes, it is GL, but overall, the GL showed the better fit for the daily returns as well as for the weekly returns.

In a nutshell, all the examinations of extremes proved that GL is a better fit for the extremes of Byco Petroleum 
TABLe 6: Daily VaR backtesting results at various confidence levels.

\begin{tabular}{|c|c|c|c|c|c|c|c|}
\hline $\begin{array}{l}\text { Confidence levels } \\
\text { Number of return }\end{array}$ & 20 & $95.00 \%$ & $97.50 \%$ & $99.00 \%$ & $99.50 \%$ & $99.75 \%$ & $99.90 \%$ \\
\hline VC250 & $\begin{array}{c}124 \\
(p \leq 0.001)\end{array}$ & $\begin{array}{c}78 \\
(0.063)\end{array}$ & $\begin{array}{c}44 \\
(0.092)\end{array}$ & $\begin{array}{c}32 \\
(0.003)\end{array}$ & $\begin{array}{c}24 \\
(p \leq 0.001)\end{array}$ & $\begin{array}{c}20 \\
(p \leq 0.001)\end{array}$ & $\begin{array}{c}16 \\
(p \leq 0.001)\end{array}$ \\
\hline HS250 & $\begin{array}{c}176 \\
(p \leq 0.001)\end{array}$ & $\begin{array}{c}92 \\
(0.089) \\
\end{array}$ & $\begin{array}{c}52 \\
(0.184) \\
\end{array}$ & $\begin{array}{c}18 \\
(0.264)\end{array}$ & $\begin{array}{c}7 \\
(0.560) \\
\end{array}$ & $\begin{array}{l}- \\
-\end{array}$ & $\begin{array}{l}- \\
-\end{array}$ \\
\hline HS1000 & $\begin{array}{c}218 \\
(p \leq 0.001)\end{array}$ & $\begin{array}{c}116 \\
(p \leq 0.001)\end{array}$ & $\begin{array}{c}50 \\
(0.173) \\
\end{array}$ & $\begin{array}{c}23 \\
(0.003) \\
\end{array}$ & $\begin{array}{c}16 \\
(0.002)\end{array}$ & $\begin{array}{c}10 \\
(0.017)\end{array}$ & $\begin{array}{c}4 \\
(0.412) \\
\end{array}$ \\
\hline MCS250 & $\begin{array}{c}140 \\
(0.059)\end{array}$ & $\begin{array}{c}83 \\
(0.052) \\
\end{array}$ & $\begin{array}{c}41 \\
(0.003) \\
\end{array}$ & $\begin{array}{c}29 \\
(p \leq 0.001)\end{array}$ & $\begin{array}{c}23 \\
(p \leq 0.001)\end{array}$ & $\begin{array}{c}20 \\
(p \leq 0.001)\end{array}$ & $\begin{array}{c}16 \\
(p \leq 0.001)\end{array}$ \\
\hline GL static & $\begin{array}{c}229 \\
(p \leq 0.001)\end{array}$ & $\begin{array}{c}144 \\
(0.007) \\
\end{array}$ & $\begin{array}{c}83 \\
(p \leq 0.001) \\
\end{array}$ & $\begin{array}{c}44 \\
(p \leq 0.001) \\
\end{array}$ & $\begin{array}{c}29 \\
(p \leq 0.001) \\
\end{array}$ & $\begin{array}{c}20 \\
(p \leq 0.001)\end{array}$ & $\begin{array}{c}2 \\
(0.829) \\
\end{array}$ \\
\hline GEV static & $\begin{array}{c}230 \\
(0.002)\end{array}$ & $\begin{array}{c}136 \\
(p \leq 0.001)\end{array}$ & $\begin{array}{c}78 \\
(0.004) \\
\end{array}$ & $\begin{array}{c}43 \\
(p \leq 0.001)\end{array}$ & $\begin{array}{c}33 \\
(p \leq 0.001)\end{array}$ & $\begin{array}{c}24 \\
(p \leq 0.001)\end{array}$ & $\begin{array}{c}12 \\
(p \leq 0.001)\end{array}$ \\
\hline GL MW & $\begin{array}{c}223 \\
(p \leq 0.001)\end{array}$ & $\begin{array}{c}116 \\
(0.002) \\
\end{array}$ & $\begin{array}{c}53 \\
(0.187) \\
\end{array}$ & $\begin{array}{c}23 \\
(p \leq 0.001)\end{array}$ & $\begin{array}{c}13 \\
(0.091) \\
\end{array}$ & $\begin{array}{c}6 \\
(0.884) \\
\end{array}$ & $\begin{array}{c}1 \\
(0.974) \\
\end{array}$ \\
\hline GEV MW & $\begin{array}{c}221 \\
(p \leq 0.001)\end{array}$ & $\begin{array}{c}115 \\
(p \leq 0.001)\end{array}$ & $\begin{array}{c}52 \\
(0.183) \\
\end{array}$ & $\begin{array}{c}23 \\
(p \leq 0.001)\end{array}$ & $\begin{array}{c}13 \\
(0.091) \\
\end{array}$ & $\begin{array}{c}7 \\
(0.610) \\
\end{array}$ & $\begin{array}{c}4 \\
(0.418) \\
\end{array}$ \\
\hline
\end{tabular}

daily returns. GL performed better than GEV in more subperiods, but in few cases, GEV went beyond GL in performance. It is also important to note that the behavior of extremes can change as the nature of the shape parameter changed with subperiods.

\section{Estimating and Comparing VaR}

EVT methods are evaluated in order to determine the efficiency of these methods to calculate VaR. Lower quantiles of extremes were used to produce VaR estimates of daily returns. To that end, parameter estimates of both GL and GEV distributions for daily extremes were put to use. Moving window method is employed on the grounds that the distribution of extremes has time-varying behavior. Traditional methods including HS, MCS, and VC were used to generate $\mathrm{VaR}$ estimates. For this purpose, 250 past daily returns were examined but for $\mathrm{HS} \mathrm{VaR}$ estimates, 1000 historical returns were used because 250 returns are fewer to calculate $\mathrm{VaR}$ at higher confidence levels. The Christofferson $p$ value is presented for all methods in parenthesis.

The results mentioned in Table 6 throw spotlight on different facts. At first, it can be noticed that the VC method overestimated the risk at lower confidence levels, but at $99.75 \%$ and $99.90 \%$, it predicted the VaR rightly where the number of violations is 20 and 16. Like the VC method, HS method also performed well at high confidence levels. However, with 1000 past daily returns, the HS method seemed to be forecasting accurately even at lower confidence levels. MCS, on the contrary, worked unsatisfying at all confidence levels. All traditional methods did not accurately measure the risk of extremes daily negative returns.

Furthermore, it can be noticed from the results of Table 5 that EVT methods based on the static approach performed badly in forecasting the risk. Only GL with the static approach worked well at the $99.90 \%$ confidence level. The reason behind this poor performance is the time-varying behavior of the distribution of extremes. So, we can see that the moving window approach eliminated this problem up to some extent. EVT methods with moving window produced accurate results at higher confidence levels. For instance, expected violations are 9, 4, and 2 at 99.50\%, 99.75\%, and 99.90\% confidence levels, respectively. However, generalized logistics distribution with the static approach resulted in 29, 20 , and 2 violations, and the outcome of GL with the MW approach is 13,6 , and 1 violations, respectively. The findings are summarized, which showed that GL and GEV performed better than HS, VC, and MCS. GL even surpasses GEV in some cases because GL has fatter tails and can evaluate larger extremes efficiently.

\section{Conclusions}

In this paper, daily returns of Byco Petroleum Pakistan Limited were investigated to determine which distribution is better able to model the risk. The whole sample and different subperiods of the daily minima were fitted by the GL and GEV distributions, and the PWM method was implied to estimate the parameters of these distributions. AD goodness-of-fit test $p$ value and parameter estimates of both distributions were presented in the tables. The findings of this paper clearly indicate that GL can adequately explain the extreme minima of daily returns. This result is important in changing the perspective regarding the poor application of GL. In EVT applications, GP and GEV are thought to be only applicable distributions, but it is evident in the conclusion that the GL distribution is a good fit for lower tail data. It can also be seen that the division of extremes into subperiods changes the nature of extremes with the passage of time.

EVT-based VaR analysis indicated that EVT methods were adequate in the risk measurement of extreme returns with lower probabilities. Among traditional methods, HS was the only method that gave accurate estimates at higher confidence levels, but the HS method needs more data points for accurate estimation as compared to other methods employed in the study. It can be noticed that GL and GEV 
were not able to estimate $\mathrm{VaR}$ at lower confidence levels because the main focus of both distributions is only on lower quantiles. After that, when the MW approach was employed, it improved the ability of GL and GEV to estimate VaR. Finally, results make it clear that applying the moving window technique helped in making the $\mathrm{VaR}$ estimates more accurate. It is recommended to expand the study by examining the efficiency of macroeconomic factors to explain the behavior of extreme distribution parameters.

\section{Data Availability}

Trading History Data (Byco Petroleum Pakistan) were used in this study. The data were taken from the State Bank of Pakistan and covered the 8-year period from January 3, 2005, to December 21, 2012.

\section{Conflicts of Interest}

The authors declare that they have no conflicts of interest.

\section{Acknowledgments}

The authors extend their appreciation to the Deanship of Scientific Research at King Khalid University for funding this work under grant number (RGP.1/26/42), received by Mohammed M. Almazah (http://www.kku.edu.sa).

\section{References}

[1] S. Aboura, "The extreme downside risk of the S\&P 500 stock index," Journal of Financial Transformation, vol. 26, no. 26, pp. 104-107, 2009.

[2] A. T. Ahmed Ghorbel, "Predictive performance of conditional extreme value theory and conventional methods in value at risk estimation," Institut Superieur de Gestion, vol. 43, no. 4, pp. 1-31, 2007.

[3] M. G. Evis Kellezi, Extreme Value Theory for Tail-Related Risk Measures, pp. 1-25, Department of Econometrics and FAME, Geneva, Switzerland, 2000.

[4] A. C. Davison and R. L. Smith, "Models for exceedances over high thresholds," Journal of the Royal Statistical Society: Series $B$, vol. 52, no. 3, pp. 393-425, 1990.

[5] M. Parkinson, "The extreme value method for estimating the variance of the rate of return," Journal of Business, vol. 53, no. 1, pp. 61-65, 1980.

[6] F. M. Longin and M. Felipe, "The asymptotic distribution of extreme stock market returns," Journal of Business, vol. 69, no. 3, pp. 383-408, 1996.

[7] E. J. Michael Rockinger, "The tail behavior of stock returns: emerging versus mature markets," Journal of Empirical Finance, vol. 10, no. 5, pp. 559-581, 2003.

[8] T. Lux, "The stable Paretian hypothesis and the frequency of large returns: an examination of major German stocks," Applied Financial Economics, vol. 6, no. 6, pp. 463-475, 1996.

[9] R. A. J. Pownall and K. G. Koedijk, "Capturing downside risk in financial markets: the case of the Asian crisis," Journal of International Money and Finance, vol. 18, no. 6, pp. 853-870, 1999.

[10] W. Morgan, J. Cotter, and K. Dowd, "Extreme measures of agricultural financial risk," Journal of Agricultural Economics, vol. 63, no. 1, pp. 65-82, 2012.
[11] G. D. Gettinby, C. D. Sinclair, D. M. Power, and R. A. Brown, "An analysis of the distribution of extreme share returns in the UK from 1975 to 2000," Journal of Business Finance \& Accounting, vol. 31, no. 5-6, pp. 607-646, 2004.

[12] K. Tolikas and R. A. Brown, "The distribution of the extreme daily share returns in the Athens stock exchange," The European Journal of Finance, vol. 12, no. 1, pp. 1-22, 2006.

[13] A. Guillou, M. Kratz, and Y. L. Strat, "An extreme value theory approach for the early detection of time clusters. A simulation based assessment and an illustration to the surveillance of Salmonella," Statistics in Medicine, vol. 33, no. 28, pp. 5015-5027, 2014.

[14] J. Chen, X. Lei, L. Zhang, and B. Peng, "Using extreme value theory approaches to forecast the probability of outbreak of highly pathogenic influenza in Zhejiang, China," PloS One, vol. 10, no. 2, Article ID e0118521, 2015.

[15] M. Thomas, M. Lemaitre, M. L. Wilson et al., "Applications of extreme value theory in public health," PloS One, vol. 11, no. 7, Article ID e0159312, 2016.

[16] B. V. Gnedenko, "Sur la distribution limite du terme d'une série aléatoire," Annals of Mathematics, vol. 44, pp. 423-453, 1943.

[17] P. Kearns and A. Pagan, "Estimating the density tail index for financial time series," The Review of Economics and Statistics, vol. 79, no. 2, pp. 171-175, 1997.

[18] M. Leadbetter, G. Lindgren, and H. Rootze'n, Extremes and Related Properties of Random Sequences and Processes, Springer-Verlag, New York, NY, USA, Springer series in statistics, 1983.

[19] E. Jondeau and M. Rockinger, "The tail behaviour of stock returns; emerging versus mature markets," Journal of Empirical Finance, vol. 10, pp. 559-581, 2003.

[20] F. M. Aparicio and J. Estrada, "Empirical distributions of stock returns: European securities markets, 1990-95," The European Journal of Finance, vol. 7, no. 1, pp. 1-21, 2001.

[21] J. Hosking, "L-Moments: analysis and estimation of distribution using linear combination of order statistics," Journal of the Royal Statistical Society, vol. 52, pp. 105-124, 1990.

[22] J. R. Hosking and J. R. Wallis, "Parameter and quantile estimation for the generalized Pareto distribution," Technometrics, vol. 29, no. 3, pp. 339-349, 1987.

[23] P. F. Christoffersen, "Evaluating interval forecasts," International Economic Review, vol. 39, no. 4, pp. 841-862, 1998. 